\title{
Philosophiques
}

\section{Texte à plusieurs voix autour d'un livre ou les suites d'une table ronde sur Le Singulier de Marc Renault}

\section{Léon Charette, René Champagne, Robert Imlay et Marc Renault}

Volume 10, numéro 1, avril 1983

URI : https://id.erudit.org/iderudit/203217ar

DOI : https://doi.org/10.7202/203217ar

Aller au sommaire du numéro

Éditeur(s)

Société de philosophie du Québec

ISSN

0316-2923 (imprimé)

1492-1391 (numérique)

Découvrir la revue

Citer ce document

Charette, L., Champagne, R., Imlay, R. \& Renault, M. (1983). Texte à plusieurs voix autour d'un livre ou les suites d'une table ronde sur Le Singulier de Marc Renault. Philosophiques, 10(1), 127-151. https://doi.org/10.7202/203217ar d'utilisation que vous pouvez consulter en ligne. 


\title{
INTERVENTIONS
}

\author{
TEXTE À PLUSIEURS VOIX AUTOUR D'UN LIVRE \\ ou les suites d'une table ronde sur Le Singulier \\ de Marc Renault
}

Lors d'un récent congrès ${ }^{1}$, trois commentateurs étaient invités à adresser des questions au livre de Marc Renault, Le Singulier. Essaie de monadologie ${ }^{2}$. La densité et la difficulté de cet ouvrage, où sont discutées les perspectives modernes sur l'interpellation de l'étant singulier et de ses intérêts par la métaphysique, ont permis aux participants de se situer eux-mêmes, en formulant interrogations et objections. Ainsi, les professeurs Léon Charette, Robert Imlay et René Champagne ont élargi le débat en choisissant de présenter dans le cadre d'une table ronde, des interventions relativement autonomes et engagées, auxquelles Marc Renault a alors répondu sur le vif. La puissance de ce livre tient en effet à sa manière de serrer de près les options premières dans tout discours théorique, et par là, de renvoyer le lecteur à l'examen des siennes. D'où la diversité des intérêts exprimés dans ces trois contributions ; elles méritent d'être considérées autant pour leurs qualités propres qu'en tant qu'explicitation de la problématique du Singulier.

Une fois le congrès terminé, l'auteur a eu l'occasion de s'arrêter sur ces commentaires qui avaient pris une forme écrite, et de rédiger une réplique. On trouvera ici les trois interventions et la réponse qui leur a été faite, pour donner une suite à ce que les échanges d'un congrès comportent de spontané et d'inachevé.

D.L.

1. Congrès de l'Association canadienne de philosophie, Ottawa, 1982.

2. Collection "Recherches« no. 22, Desclée/Bellarmin Paris/Montréal, 1979. (cf. compte rendu in "Philosophiques" IX. 2. 


\section{INTERVENTION DE LÉON CHARETTE}

Dès le début de son ouvrage sur Le singulier, l'auteur, $M$. Marc Renault, nous prévient qu'il s'agit d'un essai, et que sans vouloir faire ouvre d'historien, il s'inspire néanmoins de $M$. Maurice Blondel.

Ces précautions de l'auteur autorisent sinon encouragent celui qui n'est ni spécialiste de Blondel, ni historien de la philosophie, à ne pas recenser dans le détail les points de rencontre ou de divergence entre les deux auteurs, mais à tenter de saisir l'intention fondamentale de cette démarche qui se veut du même esprit que celui de $\mathrm{M}$. Blondel. C'est surtout de ce point de vue de l'intention fondamentale que je voudrais proposer certaines réflexions très libres et très sommaires, qui ne constituent en aucune façon un commentaire suivi des analyses magistrales et difficiles effectuées par $M$. Renault, mais qui représentent une sorte d'éventail des principaux thèmes de réflexion que la lecture de son ouvrage a provoqués dans mon esprit, de même que l'énoncé de certains prolongements possibles.

M. Renault, comme Blondel, prend son point de départ dans le problème de la destinée humaine, et veut poser le problème à partir de l'être singulier concret. Plus spécifiquement, il veut parler non seulement des conditions de possibilité de la pensée, mais encore, et peut-être davantage, des conditions de son parachèvement. Par là, $M$. Renault me semble élaborer, comme M. Blondel, une philosophe "de l'insuffisance " (pour ne pas dire de la contingence), et révéler son intention fondamentalement métaphysique. Qu'on se rappelle la remarque de Blondel :

Je n'entends ou ne lis jamais l'étiquette qu'on m'accole ‘Philosophie de l'action' sans une irritation secrète : car enfin, si j'avais publié d'abord, comme c'eût été possible et peut-être prudent pour commencer, mon étude élaborée depuis les débuts sur la Pensée ou celle sur l'Être quie je n'ai cessé de ruminer, ne m'euton pas honoré d'une autre affiche?

On ne disputera probablement pas que $M$. Blondel voulait procéder à une étude de tout ce qu'implique la finitude humaine et son inachèvement. À première vue, l'entreprise de $\mathrm{M}$. Renault me paraît à la fois plus restreinte et plus radicale : plus restreinte 
parce que centrée davantage sur la question du savoir et moins explicite sur ses propres thèses métaphysiques et épistémologique; plus radicale parce que plus globale dans sa remise en question de tout l'effort de compréhension philosophique déployé depuis Descartes, effort qui en raison de la séparation introduite entre la pensée et l'étendue est constamment guetté par la tentation de l'immanence sous une forme ou sous une autre.

Quelques textes pourraient étoffer cette brève comparaison. En ce qui concerne les ressemblances d'abord. M. Blondel écrivait dans l'Action de 1893 :

Croire qu'on peut aboutir à l'être et légitimement affirmer quelque réalité que ce soit sans avoir atteint le terme même de la série qui va de la première intuition sensible à la nécessité de Dieu et de la pratique religieuse, c'est demeurer dans l'illusion...

\section{Renault dit de manière semblable :}

Si la pensée se fragmente chez nous en pensées, il reste que dans chacune de ces pensées la loi du développement intégral de la pensée est présente : chaque moment de la progression implique la progression entière comme règle d'intégration des pensées particulières (p. 9).

Et maintenant quant aux différences : $\mathrm{M}$. Blondel écrivait dans l'Action de 1936 :

J'affirme en conséquence la vérité objective des concepts, leur contenu réaliste, l'immanence vitale en eux des réalités auxquelles ils donnent accès en notre intelligence ; et cela d'autant plus que je ne confonds pas tous les concepts en une même foule - où tous les substantifs se feraient prendre pour le vêtement d'authentiques substances - Il y a donc à opérer un triage, mais sans diminuer pour cela la portée essentielle de ce pouvoir de former des concepts ; car - il implique toujours la puissance intellectuelle qui est captatrice de vérité et d'être.

M. Renault semble moins optimiste quand il écrit :

L'étant singulier s'éveille à l'indubitable évidence qu'il y a un ordre universel de l'être, mais sans comprendre tout ce dont cette évidence enveloppante est grosse, de sorte qu'il ne sait pas comment son être s'ajuste dans le puzzle universel : quelle est la destinée d'un singulier, dont la pensée est ouverte à l'infini sur l'universelle réalité, et qui ne peut par suite se comprendre par des concepts théoriques? (p. 13) 
M. J. Maritain a traité autrefois de ce problème de la valeur des concepts par rapport à la philosophie bergsonnienne. Il raconte la chose en ces termes :

C'est en 1908, tandis que nous délibérions - si nous pouvions accorder la critique bergsonnienne du concept et les formules du dogme révélé, que l'irréductible conflit entre les énoncés ‘conceptuels de la foi théologale - et la doctrine philosophique pour laquelle nous nous étions passionnés pendant nos années d'études - nous apparut comme un de ces faits trop certains dont l'âme - sait aussitôt qu'elle ne leur échappera pas. (. . .) Il fallait choisir : il est clair qu'on ne pouvait choisir que pour l'infaillible. (. . .) Puisque Dieu nous propose dans des concepts et des propositions conceptuelles (. . .) les vérités les plus trancendantes et les plus inaccessibles à notre raison, la vérité même de sa vie divine, son abîme à lui, c'est donc que le concept n'est pas un simple instrument pratique incapable à lui seul de transmettre le réel à notre esprit, bon à morceler artificiellement des continuités ineffables, et qui laisse fuir l'absolu comme l'eau à travers un filet ... (La ph. bergsonienne, Préf. à la $2^{\mathrm{e}}$ éd. p. XIIXIII).

Le dilemme soulevé par $M$. Maritain reste posé, et il ne me semble pas, à première vue, que l'ouvrage de $M$. Renault l'ait considéré avec suffisamment d'attention. Quoi qu'il en soit de ce point, il est vrai que nos constructions théoriques resteront toujours inachevées tant en extension qu'en compréhension ; et il est peut-être encore plus facile d'admettre avec M. Renault que :

Quand l'intellectuel a dévidé le fuseau de son discours savant, il lui reste encore à penser et à faire, comme sait le faire l'homme simple. Les réfutations spéculatives de l'intériorité sont une ignoratio elenchi : elles prennent le papier pour le théorème ou la partition pour la musique (p. 89).

Sans entrer dans le détail des analyses serrées effectuées par M. Renault, il peut apparaître que c'est bien, en effet, la disparité constatée entre le savoir et le vécu concret, entre l'être et les êtres, entre la pensée et les pensées, entre le vouloir et le voulu actuel, entre le fini et l'infini, qui conduit ultimement la personne (ou monade) à la nécessité d'une option fondamentale que $M$. Renault décrit en ces termes :

ou bien la raison sera seulement l'outil technique de la possession du monde (et alors la destinée humaine se déroule entièrement 
dans la temporalité historique et naturelle), ou bien la raison ouvre effectivement un horizon nouveau, un monde spirituel transcendant, (et alors notre situation mondaine a comme sens de nous permettre d'entrer au royaume de l'esprit par consentement plutôt que d'y être mis d'office) (p. 111-112).

Ainsi, poursuit-il,

La monade tranche nécessairement cette alternative par une option qui manifeste sa volonté d'être et définit quel être elle veut (p. 115).

Mais pour donner un visage encore plus concret à cette option, peut-être pourrait-on la décrire de la façon suivante. Dans les deux cas, la personne (ou monade) choisit le transcendant, c'est-à-dire, l'autre. Mais ce choix peut se faire en deux sens possibles : par en bas, du côté de la matière conçue comme auto-suffisante, et on retrouvera soit le mythe nietzschéen de l'éternel retour, soit différentes formes d'immanentisme ou de positivisme, soit encore l'utopie marxiste de la société sans classes ; par en haut, du côté de l'esprit, on retrouvera le Transcendant au sens du tout autre et du divin.

Cette deuxième partie de l'alternative, à mon avis, peut elle-même être pressentie par une autre analyse métaphysique de l'être concret qui en laisse déjà entrevoir le projet, ne seraitce que confusément : je cite un passage de S. Thomas d'Aquin :

Quand un être trouve en un autre toute la raison de son existence et de sa bonté, il ne se peut qu'il n'aime cet autre naturellement plus que lui-même, car, - toute partie aime naturellement le tout plus que soi (. . .) Sous ce rapport tout être aime Dieu naturellement plus que lui-même (Ia Pars, q. 60, art. 5, ad. 1).

Quant au point de vue de l'origine métaphysique de l'être concret, $\mathrm{S}$. Thomas écrit encore :

Aussi longtemps qu'une chose possède l'être, il faut que Dieu lui soit présent, et cela conformément à la manière dont elle possède l'être. Or, l'être est en chaque chose ce qu'il y a de plus intime et de plus profondément inhérent, puisqu'il joue à l'égard de tout ce qui est en elle le rôle de forme, de principe déterminateur. On doit donc en conclure nécessairement que Dieu est en toutes choses, et cela de la manière la plus intime (la P., q. 8, a. 1, Resp.). 
Qui n'a pas reconnu dans cette dernière phrase un écho des Confessions de S. Augustin :

Dans ma laborieuse et haletante indigence de vérité, je vous cherchais, mon Dieu - mais vous étiez au-dedans de moi plus profondément que mon âme la plus profonde, et au-dessus de mes plus hautes cimes" (L. III, Ch. 6).

On entrevoit déjà, j’imagine, où je veux en venir par ces textes. C'est que si l'hypothèse ou l'option d'un Dieu Transcendant et personnel a du sens, les données mêmes du problème de le connaître ont totalement changé. En effet, chacun sait que celui qui est savant dans les choses morales peut se conduire d'une manière immorale, et que même l'expert en sciences religieuses peut ne pas être croyant. C'est que le savant ici parle de quelqu'un, tandis que le croyant parle à quelqu'un. Or, de même qu'on ne peut apprendre à connaître une personne seulement à penser à elle - on en resterait seulement au niveau du rêve ou de la fantaisie - de même la connaissance de Dieu ne s'acquiert pas par la simple pensée, mais par sa fréquentation (et peut-être aussi par celle de ses meilleurs amis). Il me semble donc que, au-delà de la science des savants et des philosophes, les interprètes les plus autorisés de l'expérience religieuse se trouvent chez les auteurs spirituels ou les mystiques, en qui se réalisent le plus parfaitement à mon avis la réconciliation de l'intelligence et de la volonté, l'union complète de la connaissance et de l'amour - (Henri Bergson, dans Les deux sources etc. a montré, à mon avis, l'importance de l'étude du mysticisme pour la philosophie. Et la philosophie de la religion rejoint ici la théologie mystique.) Mais, laissons ce dernier point.

Si l'on revient maintenant au point de départ de l'ouvrage de M. Renault, on se souviendra qu'il s'enracine dans le singulier. Selon les termes de l'auteur :

Nous ne pouvons pas aborder le problème philosophique là où nous ne sommes pas, dans une raison théorique détachée de la destinée concrète de chacun (p. 11).

L'auteur postulait donc l'importance et la valeur de cette destinée de l'être pensant concret. En un sens, toute la démarche de l'ouvrage consiste à vérifier cette hypothèse. Mais voilà que par l'option qui a déjà été indiquée précédemment, la monade 
ou personne peut : ou bien se choisir et s'affirmer comme autosuffisante, mais pour se perdre ultimement dans le déferlement sans fin de la matière, ou bien elle peut choisir la Transcendance divine et en un sens se perdre, mais dans l'espoir d'être ensuite rendue pleinement à elle-même. Et $\mathrm{M}$. Renault note avec raison ici :

Le consentement à Dieu ne se fait pas sans un désistement ressenti comme la mortification de notre appétit d'autonomie et de suffisance : la monade n'accepte pas d'être par l'Autre sans en rabattre d'une volonté propre qui se flatte de ne rien devoir qu'à soi (p. 117).

Peut-être est-ce là, en effet, la forme la plus haute et la plus exigeante du détachement intellectuel. Mais il avait écrit quelques lignes plus haut une phrase qui me paraît appeler certaines précisions. Je cite :

L'option pour la transcendance implique la thèse d'un droit à la singularité au sein d'un ordre universel dont la monade attend qu'il lui permettra d'être à la mesure de ses exigences idéales (id.).

Mais pour paraphraser la façon dont l'auteur voit l'existence des anges en métaphysique médiévale, "la réalité des monades est relative à la transcendance d'une liberté créatrice " (p. 108). En d'autres termes, l'existence même des monades peut bien être due à la liberté créatrice, mais leur nécessité ou permanence, leur "droit à la singularité » s'enracinent dans un autre aspect de la Transcendance, et plus précisément dans le fait révélé que Dieu est Amour.

En effet, au jugement attribué à Dieu et répété sept fois au sujet de sa création : «Dieu vit que cela était bon » (Genèse, c. i), le Livre de la Sagesse fait écho à son tour dans la prière du prophète :

Tu aimes en effet tout ce qui existe et tu n'as de dégoût pour rien de ce que tu as fait ; car si tu avais haï quelque chose, tu ne l'aurais pas formé.

Et comment une chose aurait-elle subsisté, si tu ne l'avais voulue ?

Ou comment ce que tu n'aurais pas appelé aurait-il été conservé ? (c. 11, v. 24-25) 
Dans ce contexte, il conviendrait alors tout à fait de dire que « les monades sont pensées, élues, voulues, dans leur singularité même, par le Créateur » (p. 108). C'est ultimement par l'amour gratuit dont elle est l'objet que la monade peut voir assuré et garanti son droit à la singularité. Car si aimer consiste à vouloir le bien de ce que l'on aime, comment celui qui aime pourrait-il envisager la destruction de ce qu'il aime vraiment?

Mais s'il en est ainsi, c'est alors que survient pour la monade la question soulevée par notre auteur :

Sur ce plan de l'option fondamentale, serait-il plus difficile d'ac-

cepter qu'on nous donne que de donner? (p. 117)

La question mérite en effet la plus sérieuse réflexion.

Je me rends bien compte, pour terminer, que les brefs commentaires qui précèdent relèvent, à vrai dire, moins de la critique que de l'interrogation et de l'échange. C'est pourquoi je voudrais ajouter que je sais gré à $M$. Renault d'avoir contribué par son ouvrage à alimenter la méditation philosophique personnelle de ses lecteurs.

Département de philosophie Université d'Ottawa 
INTERVENTION DE ROBERT A. IMLAY

Mon ignorance de la philosophie de Maurice Blondel est, hélas, totale. Fort heureusement, mon ignorance de la philosophie de Descartes, bien que profonde, n'est pas pour autant complète. Il me serait, par conséquent, avantageux d'aborder le très intéressant et très audacieux livre de $M$. Renault par le biais de ma connaissance des spéculations du grand philosophe français. Cela m'est d'autant plus avantageux que M. Renault en parle abondamment, si ce n'est que pour le critiquer.

Qu'est-ce qu'il reproche à Descartes au juste? Si je comprends les grandes lignes de sa critique, il lui reproche d'avoir essayé de convertir, d'une manière théorique, une philosophie de la représentation en philosophie de l'être. Une telle conversion, selon M. Renault, implique le monisme spinoziste. Mais Descartes n'est pas spinoziste et, à moins que je ne m'abuse, M. Renault non plus. Celui-là, par conséquent, toujours selon M. Renault, n'a d'autre choix que de recourir au dogmatisme pour effectuer la conversion tant désirée de la représentation en être. Et si l'on s'obstine à héberger des doutes là-dessus, $M$. Renault nous cite l'autorité de Kant, selon lequel l'être (Sein) n'est pas un prédicat réel, pour nous extraire l'assentiment ferme et inconditionnel.

Permettez-moi de dire d'abord que, l'autorité de Kant nonobstant, j'ai la témérité de ne pas être convaincu que l'existence ne soit pas un prédicat réel. Pour trancher une telle question, il faudrait d'abord une idée beaucoup plus claire, voire distincte, de ce qu'on entend par "prédicat réel ». Et ce n'est pas, que je sache, chez Kant que l'on va la trouver. Mais quand même l'existence ne serait pas un prédicat réel, je ne vois pas en quoi la conversion de la représentation en être serait nécessairement vouée à l'échec. Car quoi qu'on entende par « prédicat réel », l'imprédicabilité de l'existence ne signifierait point que je ne pusse comprendre l'être dans un concept. C'est une chose de dire que du concept à l'être la conséquence n'est jamais bonne - corollaire, il faut croire de la thèse que l'existence ne soit pas un prédicat réel. C'est une tout autre chose de dire que du concept à la comprébension de l'être, la conséquence n'est jamais bonne. Il me faudrait d'ailleurs, pour ma part, des arguments 
bien solides - je n'en vois malheureusement pas dans l'œuvre de M. Renault - avant de verser dans un scepticisme aussi irrémédiable.

Mais n'y a-t-il vraiment pas de remèdes? M. Renault parle à cette fin des intérêts pratiques de la raison. À la bonne heure ! En même temps, les intérêts pratiques ne peuvent remplir le rôle que la tradition et - oserais-je dire ? - le bon sens confient à la raison eu égard à ses intérêts théoriques. Qui plus est, les intérêts pratiques de la raison peuvent difficilement s'avérer efficaces tant qu'ils dépendront - et comment dénier une dépendance quelconque - d'une raison théorique aussi impuissante, pour ne pas dire trompeuse, que nous présente $M$. Renault.

L'un des éléments de cette prétendue impuissance de la raison théorique telle que la conçoit Descartes est, toujours selon M. Renault, son incapacité de saisir l'étant singulier. Je crainśs fort, cependant, que cette incapacité ne soit que le produit d'une interprétation abusive de la part de $\mathrm{M}$. Renault de la pensée de Descartes à ce sujet. Prenons, par exemple, le fameux cogito que M. Renault, à juste titre d'ailleurs, nomme " le socle du système cartésien ». Celui-ci, si je le comprends bien, prétend que la certitude de la pensée n'établit que l'existence d'un sujet logique. Et il faut toujours distinguer entre un sujet logique et l'étant singulier. Mais Descartes serait entièrement d'accord. Le cogito n'est point, à son avis, un exemple du principe que chaque attribut en acte, si j'ose dire, exige un sujet d'inhérence qui existe. Descartes, à vrai dire, y admet des exceptions. L'argument dit ontologique, par exemple, présuppose que l'on peut qualifier Dieu d'une certaine manière dans les prémisses sans pour autant affirmer par ce fait même qu'il existe. Sinon l'argument constituerait une pétition de principe flagrante. Or, il n'en est rien. On peut décrire des entités tout en laissant ouverte la question de leur existence. Du moins telle est la conviction de Descartes.

Mais le cogito alors? Est-ce tout simplement une autre exception au principe que chaque attribut en acte exige un sujet d'inhérence qui existe ? Si la réponse est affirmative, comment serait-il en état de prouver que Descartes existe ? Si la réponse est, au contraire, négative, comment celui-ci justifie-t-il l'ex- 
ception faite pour le cas de Dieu, tandis qu'il ne çonsent pas à faire une pareille exception pour son propre cas ? La seule façon de dénouer le nœud gordien, c'est de reconnaître que le principe en question n'a rien à voir avec le cogito. Celui-ci n'est ni une confirmation de ce principe, ni une exception à ce même principe. Il passe outre. Il s'agit d'une simple inspection de l'esprit où la pensée apparaît telle qu'elle m'appartient. Et en même temps je m'apparais à moi-même en tant qu'être singulier pour rejoindre le propos de $\mathrm{M}$. Renault - et non pas en tant que sujet logique. Descartes, autant que Blondel, accède au singulier.

Department of Philosophy

University of Toronto 


\section{INTERVENTION DE RENÉ CHAMPAGNE}

Je ferai d'abord deux remarques préliminaires :

1) Je dois avouer à ma courte honte n'avoir jamais lu les grandes œuvres de Blondel. Aussi m'est-il impossible de juger, pour cet essai dans lequel la pensée de Blondel joue un si grand rôle, ce qu'il faut rendre à Blondel, ce qu'il faut rendre à Renault.

2) J'ai lu Le Singulier après une session consacrée au marxisme. Cette session m'a poussé à ne retenir de ce livre d'une grande densité intellectuelle qu'un point précis, celui du rapport immanence-transcendance. L'immanentisme marxiste me poussait, en effet, à saisir - sous la plume de Renault ou de BlondelRenault, que sais-je ? - Comment des philosophes se trouvaient justifiés à briser le cercle de l'immanence et d'opérer le passage à la transcendance.

\section{Immanence ou transcendance ?}

Une telle question, ce n'est pas dans l'abstrait que cet essai veut la poser. Elle surgit par rapport au singulier, et, d'une façon plus précise, comme il est souvent dit, par rapport à l'intérêt du singulier que la pensée authentique ne peut jamais délaisser.

Mais de quel intérêt s'agit-il ? Ce n'est pas n'importe quel intérêt qui fera surgir la nécessité de l'option entre immanence et transcendance. La monade pensante doit élargir l'horizon de son intérêt "aux dimensions de l'intentionnalité de la raison » (82). Elle doit se hausser au niveau du désir - qui lui est spécifique - de l'intégrale réalisation de soi. " La philosophie se distingue de toute autre recherche intellectuelle en ce qu'elle adopte comme normatif de ses travaux le point de vue compréhensif de la monade concrète et singulière en qui la pensée intervient comme fonction de l'universelle assimilation du réel dans le singulier $»(89)$.

À l'égard de cet appétit d'être qui lui est propre, les aliments cosmiques de l'expérience empirique dispersée n'offrent que des «expériences apéritives» (83).

Il est plusieurs visages de l'immanentisme : 
- l'immanentisme spinozien avec sa réduction discursive de toute chose à l'unique substance et le monisme de l'idée absolue ;

- l'immanentisme positiviste qui ramène l'intérêt de la raison à ce qui est accessible à l'entendement positif et s'impose la discipline de l'agnosticisme ;

- l'immanentisme sartrien qui revendique à la fois la liberté devant les catégories de l'objectivité et le transcendant qui fonderait son être ;

- l'immanentisme idéaliste hégélien ou schellingien qui se donne un contenu par l'histoire ou par une cosmo-théogonie.

Il y a plusieurs visages de l'immanentisme, mais selon Renault-Blondel, il y a un fondement général, qualifié d'illusion volontaire aux divers immanentismes.

C'est une décision volontaire non-rationnelle qui est à la source de l'opération immanentiste. L'impératif du pouvoir pousse l'homme à vouloir une réalisation de sa destinée qui soit en ses propres mains. La théorie immanentiste vient en second lieu.

Cet impératif du pouvoir amène la raison à décréter que son intérêt et en dedans des limites de l'ordre phénoménal positif ou de l'idéalisme transcendental. Il force le jugement soit à se satisfaire du phénomène, c.-à-d., à phénoménaliser l'idée, soit à idéaliser le phénomène. L'immanentisme apparaît donc comme une mystification théorique.

Si l'option pour l'immanence n'est pas rationnelle, il nous importe de voir comment l'option pour la transcendance se présente, elle, comme rationnelle et portant le « véritable intellectualisme".

Il faut choisir car il en va du salut et de la destinée de la monade concrète et singulière ;

- ou bien nous pensons que la monade inscrit sa singularité dans un absolu transcendant ;

- ou bien nous nous commettons à l'oblitération de la monade dans une totalité moniste et immanentiste ; 
- ou bien la volonté de se suffire commande la spéculation ;

- ou bien la volonté refuse comme suffisante pour la pensée et une réalité positive inadéquate à son idée de l'universel et cette même idée fixée dans un monisme logique.

Le choix entre ces ou bien . . . ou bien . . . ce sont les exigences inscrites dans la nature de la monade pensante qui le commandent et l'orientent.

Dans l'opération immanentiste, il y a un rabais quant à soi-même. La monade, dans une telle option, porte sur ellemême un verdict décisif quant à sa capacité d'être le singulier universel dont elle a l'idée. Elle n'accorde pas crédit à ses exigences rationnelles. Elle refuse la possibilité de réalisation de ce dont elle a l'idée.

L'immanentisme, quel qu'il soit, a pour loi de " gommer d'une manière ou d'une autre, la singularité de l'étant » (107).

Il conduit soit à un retrait spinozien, réducteur du singulier, soit à un phénoménisme du sujet sans cohérence et voué à l'absurde, soit à une systématique idéaliste ignorant le sujet. Il conduit à la schizophrénie ou du sujet sans raison ou de la raison sans sujet.

Opter pour la transcendance, c'est proclamer, au contraire, les droits de la singularité au sein d'un universel dont la monade espère qu'il la comblera.

On ne peut qu'être sensible à l'attention que cet essai de monadologie apporte au sort de la monade concrète et singulière. C'est bien en effet au nom de cet intérêt pour la monade pensante que se fait le passage à la transcendance.

Il y a trois questions que je voudrais ici poser à monsieur Renault, questions qui renvoient à mes remarques du début.

1) Est-il bien sûr que tous les immanentismes reposent sur un impératif du pouvoir? Si l'on songe à Hegel, on soulève un problème immense, pour ne citer qu'un exemple.

2) Ilm'apparaît qu'il y a dans le livre de Marc Renault un non-dit en ce qui touche la transcendance. De mystérieux chu- 
chotements accueillent la monade pensante dans sa démarche philosophique. En vertu de quoi, est-on enclin à demander, la monade qui opte pour un absolu transcendant opte pour sa propre transcendance par rapport à la totalité immanente ? Tout comme il n'est pas dit de quelle nature est la réalisation de la monade singulière, iln'est pas dit de quelle nature est cette transcendance qui permet la réalisation.

3) Il est souvent question dans ce livre de la monade concrète et existante. Mais est-ce vraiment le cas ? De quel secours peut être le blondélisme pour tant d'êtres concrètement engagés dans un labeur quotidien opprimant, aux prises avec des déterminismes sociaux et économiques, incapables de se hausser au désir de la réalisation intégrale de soi et, ainsi, à la transcendance, si ce n'est à une transcendance aliénante, au sens de Marx?

Il n'est pas sûr dans un tel cas que ce soit l'impératif du pouvoir qui maintiene dans l'univers clos de l'immanence.

Y a-t-il dans le blondélisme une réflexion sur les aspects sociaux et économiques soulignés par Marx et pour leur intégration sensée dans le passage de l'immanence à la transcendance?

Département de philosophie Université de Sudbury 


\title{
RÉPONSE AUX COMMENTAIRES DE MM. LÉON CHARETTE, ROBERT IMLAY ET RENÉ CHAMPAGNE
}

par Marc Renault

\author{
I
}

Le professeur Imlay note que Descartes est très présent dans Le Singulier, mais semble trouver qu'il y est trop uniquement objet de critiques. Cela prouve combien j'ai été malhabile, puisque la présence des philosophes dans cet ouvrage devrait témoigner d'un endettement et d'une gratitude à leur égard. Il y a bien peu de pensées originales dans Le Singulier. Cependant, quand on se propose de faire autre chose que la seule histoire de la philosophie, quand on veut s'approprier la pensée de ces philosophes pour lesquels on éprouve quelque chose comme de l'amour, on frappe des limites, on constate des points de rupture. Cette impossibilité d'épouser intégralement la pensée d'un philosophe admiré, c'est mon échec à son égard, et les critiques formulées sont l'autopsie de cet échec. Cela ne concerne pas Descartes, ou Spinoza, ou un autre, en eux-mêmes. Cela me concerne en tant que je ne peux pas être cartésien, spinozien, etc, jusqu'au bout. C'est la seule façon d'éviter l'outrecuidance d'avoir adéquatement compris ces philosophies inépuisables. On peut seulement répondre de ceci devant une grande philosophie : partant de la compréhension qu'on en a au moment où l'on parle, on présume d'abord l'adhésion et on ne se résout à lever cette présomption que là où elle crée une contradiction avec soi-même. Ce point de rupture change à mesure que l'on pénètre plus loin dans la pensée d'autrui, à mesure aussi que soi-même, on change. Et ces deux " mesures" sont peut-être la même . . ., de sorte qu'il est peut-être toujours trop tôt pour prendre position. De toute manière, on n'entre pas dans une grande philosophie sans consentir à être changé par elle, à quelque degré, entre un point 
de rentrée et un point de rupture. Et même, à un niveau plus humble, quel professeur niera qu'il est changé, parfois, par la copie d'un élève?

II

Le professeur Charette trouve dans Le Singulier des symptômes de dévaluation de la pensée conceptuelle, alors que, par ailleurs, mon texte n'offre pas grand-chose comme justification. Le professeur Imlay se demande à son tour comment on peut éviter le scepticisme quand on n'admet pas le passage « du concept à la comprébension de l'être. " - Tout ce qui est pensé dans un concept peut se trouver en position de prédicat dans une proposition. Pour l'être (esse), par contre (et c'est le sens de la remarque de Kant : Sein ist offenbar kein reales. Prädikat), seule la position de sujet est possible. Les questions conceptuelles concernent la prédication. Les questions d'être concernent la référence. Il est vrai que dans les mouvements spontanés de la pensée, nous allons et venons sans cesse entre ces deux ordres, mais pas au hasard, car nous savons placer l'étant en position de sujet quand les autres termes sont de simples concepts. La pensée finie et discursive ne peut affirmer l'être que d'une façon relative, à partir de soi. En conséquence, dans un «c'est ", il y a des médiations transcendantales; temps, espace, concepts, idées. Ces médiations ne sont des réceptions (temps-espace), des constitutions (concepts) et des règles (idées) que dans l'ordre de la possibilité, pas dans l'ordre de l'être. En particulier, le concept constitue en subsumant et cette subsomption ne gouverne que la possibilité générique de ce qu'elle subsume. Donc, pas de compréhension de l'être par les a priori du pouvoir de connaître (rupture avec Heidegger qui accorde un privilège à l'a priori du temps). Pas de compréhension, mais structuration de la référence de la pensée à l'être. Les a priori donnent à la pensée de pouvoir constituer un système immanent et cohérent de ses exigences par rapport à l'être. Et selon la manière dont chacun élabore ce système, il y aura différents discours ontologiques. L'important est de noter comment il est impossible d'éviter soit la cristallisation du système immanent (ordre de la prédication) soit de s'en servir dans des jugements d'être (ordre de la référence). De cette manière, on est loin, me semble-t-il, de déprécier la 
pensée conceptuelle puisqu'on lui attribue un rôle autrement plus décisif que dans tous les systèmes où l'être est de quelque manière ramené à la compréhension des concepts. Et l'on n'est pas sceptique, puisque concepts et idées sont cela même sur quoi la pensée prend appui pour dire oui ou non à l'être. Je laisse au bergsonisme la responsabilité de sa critique du concept, mais dans une perspective blondélienne "les énoncés conceptuels de la foi théologale " (pour reprendre le mot de Maritain cité par M. Charette) sont loin d'être négligeables : ils sont la représentation d'une médiation décisive, inévitable, entre le croyant tel qu'il peut connaître dans sa situation historique et le Dieu transcendant tel qu'il échappe à toutes les conditions de possibilité de l'expérience et de la connaissance humaines. Dans l'hypothèse d'un rapport où c'est Dieu qui prend l'initiative, il importe que soient données au croyant les structures immanentes qui lui permettent de faire retour d'une manière qui s'accorde avec les intentions de Dieu à son égard. Or, dans ces structures, il y a des représentations conceptuelles, inévitablement, dont les humbles et les petits se servent aussi bien que les savants. Rien n'empêche, bien sûr, que les savants isolent ces concepts pour les fixer en énoncés dogmatiques ou en thèses de théologie spéculative, mais on les mutile dans leur vérité si l'on croit que c'est en cela qu'ils sont vrais, c'est-à-dire si l'on pense que c'est leur vérité que d'être un énoncé dogmatique ou une thèse théologique. Si cela était exact, la vérité qui sauve serait réservée au seul théologien. Dans les controverses entre néo-scolastiques et Blondel, qui a davantage magnifié le concept, sinon Blondel, puisqu'en l'insérant dans le devenir effectif de chacun, il lui accorde l'importance la plus décisive dans l'accomplissement de la destinée humaine ? Est-ce déprécier le concept que de mettre en lumière comment notre jugement sur sa valeur de vérité contient une option sur ce que nous espérons être ? Je préférerais l'étiquette d'agnostique (celui qui ne sait pas) à celle de sceptique (celui qui en reste à l'examen des choses), car c'est mon propos d'amener la réflexion à ce moment où l'on sait que rien du savoir et de la connaissance ne peut suppléer à l'action et à l'engagement pratique. Oui, nous connaissons quelque chose dans le concept : nous connaissons qu'il est la médiation vraie qui nous ouvre sur un inconnu. Il est la science de notre non-connaissance, science qui nous met en demeure de prendre une décision envers ce que 
nous ne connaissons pas. Et cette science est au moins assez éclairante pour nous rendre responsable de cette décision. Cela laisse loin derrière les concepts de Maritain dont la vérité est trop fragile pour devenir un ingrédient de "l'effectuation " de la destinée humaine, puisqu'elle doit être pesée dans la balance d'un intellect spéculatif séparé de l'usage que nous faisons de notre liberté. - Cette réponse est une ébauche. Il faudrait aussi situer le concept dans l'ensemble de l'analogie de l'être. Une autre fois.

III

Il ne serait pas exact de dire sans nuances que le cartésianisme est une philosophie de la représentation. D'abord le cogito s'affirme être, et ensuite le système mise sur l'être de Dieu comme fondement de sa vérité. C'est déjà beaucoup d'engagement dans l'être avant même que ne démarre le système théorique du savoir. Reste que sur ce socle ontologique, le discours cartésien se déroule comme représentation dans la substance pensante. Les réalités objectives de la représentation sont autant de déterminations appartenant à cette substance. Ainsi Descartes peut-il construire sa physique en entier sans avoir à trancher la question de la réalité formelle des corps. Voilà ce que l'on pourrait appeler la "forme " spinozienne du cartésianisme : une substance pensante et tout un monde de déterminations qui sont les prédicats de cette substance. En invoquant le Dieu créateur, Descartes s'efforce de briser la limite de ce monde objectif de la représentation pour déboucher sur la réalité formelle, mais cet effort prend appui sur un seuil que Spinoza choisira de faire sien, sans essayer, comme Descartes, de l'outrepasser en convertissant des représentations en substances (ce que Kant appelait « idéalisme rêveur »). Le prof. Imlay pense que je suis contre une telle conversion, c'est-à-dire contre le passage de la prédication à la référence. Oui et non. Avec Kant, je pense qu'une telle conversion n'est pas fondée théoriquement et qu'elle ne relève pas d'un savoir. Par contre, je pense que nous faisons cette conversion ou refusons de la faire, car c'est un fait que dans ce que nous pensons nécessairement, il y a quelque chose qui nous reste à vouloir ou à refuser, et que cette détermination volontaire prise par rapport à la représentation change notre manière d'être. Cela 
implique que la philosophie de l'être n'est pas un savoir théorique, et aussi qu'il n'est pas nécessaire d'être philosophe patenté pour en avoir une. Y a-t-il moyen d'expliquer autrement comment un idéaliste et un matérialiste, un sceptique et un dogmatique, un athée et un croyant en Dieu, peuvent se parler et se comprendre? Mais cette compréhension, bien sûr, en reste au palier des vouloirs possibles. Il y a une autre communication, plus haute, qui présuppose une concorde des vouloirs effectifs, mais dans notre monde éclaté, les philosophes doivent le plus souvent se contenter d'échanger des possibles.

\section{IV}

Le professeur Charette se glisse avec aisance entre les lignes du Singulier, mais non sans les faire bouger un peu . . . Il souligne les implications religieuses de l'ouvrage. Cet aspect est volontairement atténué dans le texte, afin que l'option religieuse reste, dans le discours philosophique, simplement une possibilité pensée par la raison autonome, et une proposition faite à la liberté. À la limite, il s'agirait de rendre manifeste qu'une idée comme celle de "salut ", telle que véhiculée dans la tradition chrétienne, est nécessairement pensée comme possible, même si le salut ne peut être sinon par don gratuit de Dieu. À cette condition, la foi ne serait pas une aliénation, car elle pourrait s'appuyer sur l'a priori immanent de sa possibilité. Il appartient à la philosophie de penser analytiquement à ce salut possible qui ne sera considéré comme effectif que dans l'acte synthétique de la foi prononçant que c'est. Ce passage du prédicatif à la référence marque le moment où le philosophe sort de son système pour en disposer, ou au contraire choisit de rester dedans comme si le système devait disposer de lui. Et il appartient aussi à la philosophie de mettre en lumière cette alternative, non pour démontrer théoriquement quel parti est à prendre, mais pour faire voir qu'on ne peut éviter de prendre parti.

Quand le professeur René Champagne ne sait trop s'il a affaire à Blondel ou à Renault, cela montre que je ne sais pas encore où se trouve le point de rupture à partir duquel Blondel deviendrait un lieu de comparaison extérieur, une 
référence parmi d'autres dans l'histoire de la pensée. Cette ambiguïté ne me gêne pas, pourvu que le lecteur sache qu'il n'y a pas d'a priori méthodique de m'en tenir à une interprétation de Blondel pour lui-même. L'analyse de $\mathrm{M}$. Champagne fait ressortir que Le Singulier est une prise de position ou une espèce de manifeste philosophique. C'est juste. Et d'emblée il met le doigt sur un point névralgique de cette position, à savoir que le discours théorique d'un philosophe n'est pas ce par quoi il se trouve une position, mais ce par quoi il trouve quelle est la position qui lui donne de quoi penser une théorie. C'est mon postulat que, de toute manière, il y a une prise de position chez celui qui se met à philosopher. Elle se coagule au centre de la tornade des désirs, des vouloirs, des pensées et des actions. On sent d'abord cette masse comme un dä̈môn socratique qui incline les jugements dans un sens défini. L'analyse réflexive vient ensuite et le discours qu'elle produit a pour office, non de constituer, mais d'élucider ce qui se constitue à ce point de convergence singulier qui fait du discours philosophique une parole à la première personne : Je. Or ce Je est si peu une évidence théorique sûre de soi, que la théorie peut revenir sur lui et l'expliquer comme une apparence produite par des conditionnements qui sont, eux, exactement déterminables et doivent donc être pris pour la réalité vraie. Depuis la Critique de Kant, on devrait savoir qu'une telle opération consiste à prendre les représentations théoriques d'entendement pour les tourner vers une idée de la raison afin de la réduire. Mais comment se fait-il que les règles idéales de l'usage de l'entendement théorique deviennent ainsi victimes des théories? Il n'y a aucun savoir qui puisse fonder une telle «autophagie ». Je sais bien aussi qu'il n'y a pas de savoir pour fonder ma position, mais en optant pour une einaïlogie (néologisme pour éviter ontologie) du Je, j’aligne la volonté sur la possibilité idéale d'un singulier fondé, tandis que la dissolution du singulier dans le savoir aligne cette possibilité idéale sur la volonté de tenir toute réalité dans l'immanence du système théorique, avec la conséquence que le jugement d'être est contraint de s'arrêter à court des possibilités que lui ouvre la raison. Ces options divergentes ne nous rendent ni plus savants ni plus ignorants, mais elles sont une confiance ou une défiance envers la rationalité : confiance que l'on peut être à la mesure de l'idée, défiance envers une idée qu'il faut faire rentrer dans le rang par 
une explication immanente, afin qu'elle ne nous égare pas en ce qui transcende notre connaissance. La confiance conteste la souveraineté de la théorie. La défiance a besoin que la théorie soit souveraine. Ceux qui accordent le plus à la théorie accordent moins à la raison. La volonté intervient d'un bord comme de l'autre, mais il me semble que l'on est plus respectueux de la rationalité avec une volonté qui mesure son consentement einaïlogique sur la possibilité de l'idée, qu'avec une volonté qui somme l'être de rester dans les limites de ce qui peut être déterminé par entendement. L'immanentisme est une restriction de la portée de l'idée, restriction par laquelle la monade singulière cesse d'échapper au savoir puisqu'elle implique la croyance qu'il n'y a pas de monade.

Dans sa première question, $M$. Champagne demande s'il est « bien sûr que tous les immanentismes reposent sur un impératif de pouvoir ». Il a noté que j'attribue cette option immanentiste à un tel impératif, puisqu'en ramenant la réalité à sa compréhension dans le savoir on la met à la portée de la domination volontaire. L'immanentisme me paraissait comme le miroir du totalitarisme : rien n'échappe à la volonté là où le savoir est la compréhension adéquate de ce qui est. À la réflexion, je me demande s'il n'y a pas encore un autre type d'immanentisme, celui d'un singulier qui se désisterait de son désir d'être, sans pour autant se donner une justification théorique totalitaire, et cela précisément parce qu'il aurait compris comment le savoir totalitaire se contredit en excluant la réalité du principe qui le produit, à savoir la volonté dominatrice du théoricien. À celui qui serait ainsi brouillé avec le savoir dominateur et qui ne s'aimerait pas assez pour faire crédit à l'idée d'une identité singulière fondée sur un principe transcendant, il resterait la voie du nihilisme esthétique où il s'attribuerait assez de réalité pour se sentir vivre, mais pas assez pour que la succession temporelle de son expérience soit cohérente, et constitue la gestation d'une destinée à la mesure de l'idée monadologique. Pour le nihiliste, le temps et l'histoire ne sont pas une genèse, ni une montée vers la lumière, mais une destruction et un obscurcissement. Et l'existence est une apparence rejetable quand son spectacle n'offre plus d'attrait. - Enfin, M. Champagne avance le " problème immense » qu'est l'exemple de Hegel. C'est trop pour moi, mais 
je lui retourne son problème avec une hypothèse : est-ce qu'on ne tiendrait pas un symptôme de pensée immanentiste chaque fois que, à la suite de Hegel, les intellectuels des 150 dernières années ont ramené les questions d'éthique à des questions de culture ? Quant à Schelling, je ne l'accouplerais plus avec Hegel comme je l'ai fait à la page 117 .

Deuxième question : là où $\mathrm{M}$. Champagne perçoit des chuchotements, M. Charette avait entendu des paroles assez distinctes. Je me permets de renvoyer au paragraphe IV ci-dessus. Ce sera au moins un commencement de réponse pour une question qui perdrait son sens si elle recevait sa solution adéquate dans le système même.

La troisième question formule un doute sur la prétention de s'occuper "de la monade concrète et existante " dans un discours comme Le Singulier qui semble planer au-dessus des oppressions très concrètes de la vie quotidienne, au-dessus des déterminismes sociaux et économiques, et n'apporte ainsi aucun «secours » à la piétaille humaine où se trouvent, en définitive, les singuliers. Cette question est l'effet d'une générosité que j'apprécie depuis longtemps chez mon collègue Champagne. Si l'on se place du point de vue du contenu conceptuel de mon texte, la question manifeste fort à propos un vide : je sais bien qu'il y a tout un continent de conditionnements immanents non explicités dans mon manifeste. Cela n'a rien à voir avec les lacunes du blondélisme, mais concerne seulement mes limites. Donc, oui, si l'on cherche les prédicats qui feront connaître quelles sont les aliénations à surmonter, la récolte est mince et je ne chercherai pas à m'en excuser. Cependant, la pertinence d'un discours philosophique pour une monade singulière dépend-elle de son degré de richesse conceptuelle et de l'exhaustivité de ses analyses? Le sens global de mon plaidoyer était de revendiquer le droit pour la monade de n'être jamais réduite à l'état de prédicat dans le système afin qu'elle demeure irréductible comme sujet auquel appartient le système. Et alors je pourrais retourner la question modifiée à son auteur : pense-t-il que pour se réaliser effectivement la monade a besoin du « secours » de tous les concepts qui nomment ses conditionnements (Un « secours», la philosophie ?) Le philosophe qui pourrait nommer toutes les aliénations n'aurait aucun privilège décisif par rapport aux autres 
s'il est vrai que l'identité de la monade ne dépend pas de son habileté à se donner une justification théorique de sa transcendance par rapport à ses déterminismes. C'est dans les actions dont elle est le sujet que la monade est un singulier fondé ou qu'elle ne l'est pas. Rien ne supplée à l'action, mais l'action peut suppléer aux déficiences des analyses qu'on en fait, puisqu'elle ne tire pas sa réalité de ces analyses. La volonté n'a pas besoin de savoir tout ce qu'elle veut pour vouloir effectivement tout ce qu'elle est, comme dirait Blondel. Sans que cela me serve d'excuse pour ne pas avoir " désimpliqué " les très réelles médiations dont mon collègue fait état, je crois que d'autres experts en d'autres niveaux d'analyse pourraient eux aussi trouver d'autres continents conceptuels laissés à l'état implicite dans mon très mince essai.

Que mes trois collègues soient remerciés pour leurs interventions bienveillantes et stimulantes. Quelle que soit la qualité de cette discussion, le crédit pour sa réalisation revient au professeur Danièle Letocha qui en a eu l'idée et qui l'a organisée.

Département de philosophie

Université du Québec à Trois-Rivières

Le 6 septembre 1982 